\title{
La notion de programme et de modèle d'enseignement en langue de spécialité
}

\section{Claude Sionis}

\section{(2) OpenEdition}

\section{Journals}

Édition électronique

URL : http://journals.openedition.org/asp/4040

DOI : $10.4000 /$ asp. 4040

ISSN : 2108-6354

\section{Éditeur}

Groupe d'étude et de recherche en anglais de spécialité

\section{Édition imprimée}

Date de publication : 1 décembre 1994

Pagination : 97-110

ISSN : 1246-8185

\section{Référence électronique}

Claude Sionis, "La notion de programme et de modèle d'enseignement en langue de spécialité », ASp [En ligne], 5-6 | 1994, mis en ligne le 05 décembre 2013, consulté le 19 avril 2019. URL : http:// journals.openedition.org/asp/4040 ; DOI : 10.4000/asp.4040

Ce document a été généré automatiquement le 19 avril 2019

Tous droits réservés 


\title{
La notion de programme et de modèle d'enseignement en langue de spécialité
}

\author{
Claude Sionis
}

\section{Introduction}

1 Guides indispensables pour les uns, carcans intolérables pour d'autres, vagues et lointaines références pour la majorité des enseignants, les programmes officiels d'enseignement des langues ont au moins le mérite d'exister et rares sont les professionnels qui en ignorent totalement les grandes orientations. Ceci vaut évidemment pour les langues à usage général et essentiellement pour l'enseignement secondaire.

2 Pour ce qui est des langues dites de spécialité ${ }^{1}$ qui sont majoritairement enseignées audelà du baccalauréat dans les IUT et les Universités, et au-delà des classes préparatoires dans la plupart des grandes écoles, la notion de programme, même propre à un seul projet de formation, est souvent absente. Le praticien de LDS a, en effet, toujours été, par nécessité, un créateur ou un découvreur de matériaux d'enseignement mais pas forcément un concepteur de programmes.

3 Si finalement peu d'enseignants de langue générale doutent de l'utilité de suivre un programme, aussi ténu soit-il, il n'en est pas forcément de même en LDS. Dans ce domaine, nombre de cours sont dépourvus de logique de progression interne ou bien sont liés à une mince ligne directrice structurale ou lexicale sur laquelle se greffent des textes dits « spécialisés » provenant en fait souvent de la presse généraliste. Celle-ci est, en effet, la seule qui soit accessible, en tout cas en début de carrière, à un professeur de LDS dont la propre formation relève souvent des seules « humanités ».

4 Il est vrai qu'il n'est pas toujours aisé de trouver un cap pédagogique en LDS et que le professeur isolé ou occasionnel n'a pas toujours les moyens de se livrer à une analyse des besoins qui, pour être rigoureuse et efficace, exige durée et effort soutenu. Le professeur 
de langue générale à qui incombe pour la première fois la charge de former des apprenants à une LFP a souvent le choix entre deux stratégies pédagogiques.

5 La première équivaut à la transposition d'une langue dans une autre d'un cours spécialisé en droit, médecine, science, technologie, etc. La seconde consiste à consulter ses collègues spécialistes du domaine d'application, à se plonger dans la lecture des publications appartenant à ce domaine et à constituer des corpus de production. Ces documents seront ensuite utilisés comme autant de matériaux d'enseignement et de modèles vers lesquels les apprenants devront tendre. Cette démarche est le pendant en LDS de celle qui consiste, en langue générale, à attendre des apprenants qu'ils puissent égaler des locuteurs natifs.

6 Le professeur qui aura adopté cette seconde stratégie aura choisi la voie du cours spécifique au domaine d'application, mais il existe d'autres voies en LDS.

7 C'est l'une de ces voies qui a été empruntée par Hutchinson et Waters (1980). À l'issue de leur consultation avec les professeurs scientifiques et techniciens de leurs élèves, ces linguistes ont découvert que « la question n'était pas de savoir si le contenu du domaine du génie mécanique était différent de celui des télécommunications, mais si l'étude de textes appartenant au génie mécanique était le meilleur moyen de préparer un étudiant à un cours de génie mécanique» (notre traduction). Aucune théorie de l'apprentissage communicationnel des langues ne prescrit, en effet, que les aptitudes à acquérir dans un contexte spécialisé doivent l'être en ayant recours à ce contexte spécialisé.

8 Comme la dernière partie de l'article essaiera de le démontrer, la thèse proposée est non seulement celle selon laquelle l'apprentissage communicationnel d'une LDS bénéficie du recours à des contextes autres que ceux relevant du strict domaine d'application, mais aussi celle qui prétend que c'est lorsque le programme d'enseignement affiche ouvertement des objectifs autres que strictement linguistiques que certaines aptitudes discursives et formes linguistiques peuvent s'acquérir durablement.

9 La notion de programme devra être comprise ici comme l'organisation des matériaux pédagogiques et des cheminements méthodologiques en fonction de besoins et contraintes en cours d'apprentissage et d'un niveau de compétence en langue cible. Ce niveau devrait être idéalement déterminé à l'issue d'une analyse des besoins et des moyens au sens le plus large possible. Il convient, en effet, de prendre en compte les besoins des apprenants, ceux des contextes langagiers qui les solliciteront et des institutions qui les emploieront dans le cas des LFP, ou les besoins et moyens du cadre d'apprentissage de l'établissement d'origine et de celui d'arrivée dans le cas des LEE. Les nécessités, manques, désirs et motivation des apprenants sont également à intégrer à toute démarche organisationnelle du cours de LDS, comme nous invitent à le faire Hutchinson et Waters (1987 : chap.6).

10 L'article prendra comme axiome qu'il est de l'intérêt de tout projet d'enseignement d'une LDS de se réclamer d'un modèle théorique et d'un mode d'organisation pratique, ne serait-ce que parce que ce nouveau domaine de l'enseignement des langues se trouve, plus que tout autre, dans l'obligation de "rendre des comptes", souvent au sens premier du terme, à ses commanditaires, son public ou ses structures d'accueil.

11 L'article évoquera donc l'évolution de la notion de programme en LDS. Un premier stade sera l'adoption pure et simple de démarches organisationnelles s'appliquant originellement à des langues générales. Le stade suivant verra apparaître des conceptions hybrides, ou de transition, plus adaptées, et sera suivi lui-même par des démarches 
spécifiques conçues d'emblée pour les LDS. Cette évolution nous amènera à une proposition d'organisation qui rompe avec la conception linéaire d'un programme reposant sur une continuité ou une gradation des formes linguistiques ou discursives à acquérir.

\section{Démarches de langue générale}

12 L'un des buts de tout programme d'enseignement est de fractionner la masse de savoir et de savoir-faire en unités enseignables et susceptibles d'être appropriées durablement par l'apprenant.

13 Comment, dès lors, allier la satisfaction de besoins linguistiques, comportementaux, professionnels ou académiques, les exigences du contexte d'apprentissage et les attentes du public des apprenants?

14 À l'opposé d'une conception trop strictement spécifique de l'organisation du cours de LDS qui aurait fait passer celui-ci de l'état d'enseignement à celui d'entraînement (cf. Widdowson, 1983: 16), de nombreux praticiens de LDS se sont tournés, au début des années 1970, vers un mode de programmation du cours directement inspiré de celui emprunté par l'enseignement des langues à usage général.

15 L'une des conceptions traditionnelles les plus clairement formulées est sans doute celle de Mackey (1965). Ce linguiste pose que toute démarche organisationnelle en enseignement, et toute «méthode » dans le sens de stratégie pédagogique de la part de l'enseignant, comprend successivement les quatre composantes que sont la sélection, la gradation, la présentation et la répétition.

16 Ces quatre éléments, présentés sur le même plan par Mackey, nous semblent cependant ressortir de deux catégories conceptuelles différentes : si la sélection et la gradation des matériaux et des activités du cours relèvent d'a priori idéologiques et d'options méthodologiques générales, la présentation et la répétition font partie, quant à elles, de la routine et des techniques de classe. Cette distinction n'enlèverait rien à la pertinence de l'observation de Mackey si le fait de poser chronologiquement l'antériorité de la sélection et de la gradation ne les faisait apparaître comme indispensables à tout programme d'enseignement.

17 Ceci signifie que les choix quantitatifs et qualitatifs des formes linguistiques à acquérir peuvent se faire de manière totalement prévisible et selon des critères déterminés et reproductibles dans tous les cas. Mackey préconise, par exemple, que la sélection des items linguistiques obéisse immanquablement à quatre grands ensembles de facteurs de choix qui se déclinent eux-mêmes en un certain nombre de sous-facteurs (cf. tableau 1).

Tableau 1

\begin{tabular}{|l|l|l|l|}
\hline $\begin{array}{l}\text { Sélection } \\
\text { phonétique }\end{array}$ & $\begin{array}{l}\text { Sélection } \\
\text { grammaticale }\end{array}$ & $\begin{array}{l}\text { Sélection } \\
\text { lexicale }\end{array}$ & Sélection sémantique \\
\hline & structures & noms concrets & significations lexicales \\
\hline & inflexions & noms abstraits & $\begin{array}{l}\text { significations } \\
\text { structurales }\end{array}$ \\
\hline
\end{tabular}




\begin{tabular}{|l|l|l|l|}
\hline & mots structuraux & «modificateurs » & \\
\hline & & verbes & \\
\hline
\end{tabular}

L'idée même selon laquelle la langue à enseigner peut être prévue, quantifiée et répertoriée a priori et avant que le cours ne débute véritablement nous semble hasardeuse en LDS. Il serait certainement souhaitable de pouvoir cerner de manière rigoureuse et exhaustive toutes les formes linguistiques dont seraient redevables des apprenants non spécialistes de langues et dont les disponibilités exprimées en heures de cours sont souvent mesurées.

Il est vrai aussi que le cours de LDS est souvent jugé à l'aune de la pertinence et de la sélection rigoureuse des objectifs, mais la pertinence oblige souvent le professeur à recourir à des documents dits "authentiques » (c.-à-d. non destinés originellement à l'enseignement) et dont les contenus se prêtent mal à une sélection et à une gradation des formes qu'ils véhiculent avec une grande variété de niveaux de difficulté et d'intérêt au sein du même support.

Si l'on superpose les nombreux autres critères que mentionne Mackey pour le choix des matériaux et des exercices à proposer aux apprenants, peu de textes authentiques ou non échapperont aux filtres successifs que sont :

1) les choix de type de langue : dialecte, registre, style, medium (oral ou écrit)

2) les choix quantitatifs :

a) externes : intention, niveau, durée, type

b) internes: nombre, composabilité, information, probabilité, combinabilité, structuration.

3) les choix des critères : fréquence, portée, disponibilité, recouvrement, «learnability » (capacité à être appris), etc.

Pourtant certains critères sont essentiels en LDS, comme ceux tenant au type de langue à enseigner tels les registres (scientifique, juridique, commercial, etc.) ou le style (littéraire, familier, standard, populaire, etc.) ; mais comment isoler dans un seul support, et sans le dénaturer, l'illustration d'un nombre réduit d'éléments afin de rendre possible leur apprentissage ? Si cela était possible, le nombre de supports devant être inclus dans le cours pour pouvoir illustrer tous ces paramètres devrait être très élevé. Quel rôle écrasant ne devrait-on alors faire jouer à la phase de «présentation » de ces formes pour les rendre assimilables, et quelle quantité de « répétition » devrait être prévue dans le cours pour en fixer l'assimilation?

\section{Démarches hybrides ou de transition}

Bien d'autres modèles méthodologiques en matière de conception du déroulement d'un cours de langue générale ont été sollicités pour guider l'enseignement d'une LDS. La plupart d'entre eux tenaient compte à la fois des exigences propres à l'apprentissage d'une langue et du fait que cet apprentissage se déroulait le plus souvent en milieu institutionnel. En fait, chaque étape dans l'évolution du concept de programme d'enseignement ajoutait une dimension ou un ensemble de paramètres supplémentaires. 
Ceux-ci permettaient de mieux prendre en compte différents critères de choix conditionnant la méthodologie, le déroulement du cours et le choix des matériaux.

Fig. 1 Modèle du processus d'enseignement/apprentissage des langues

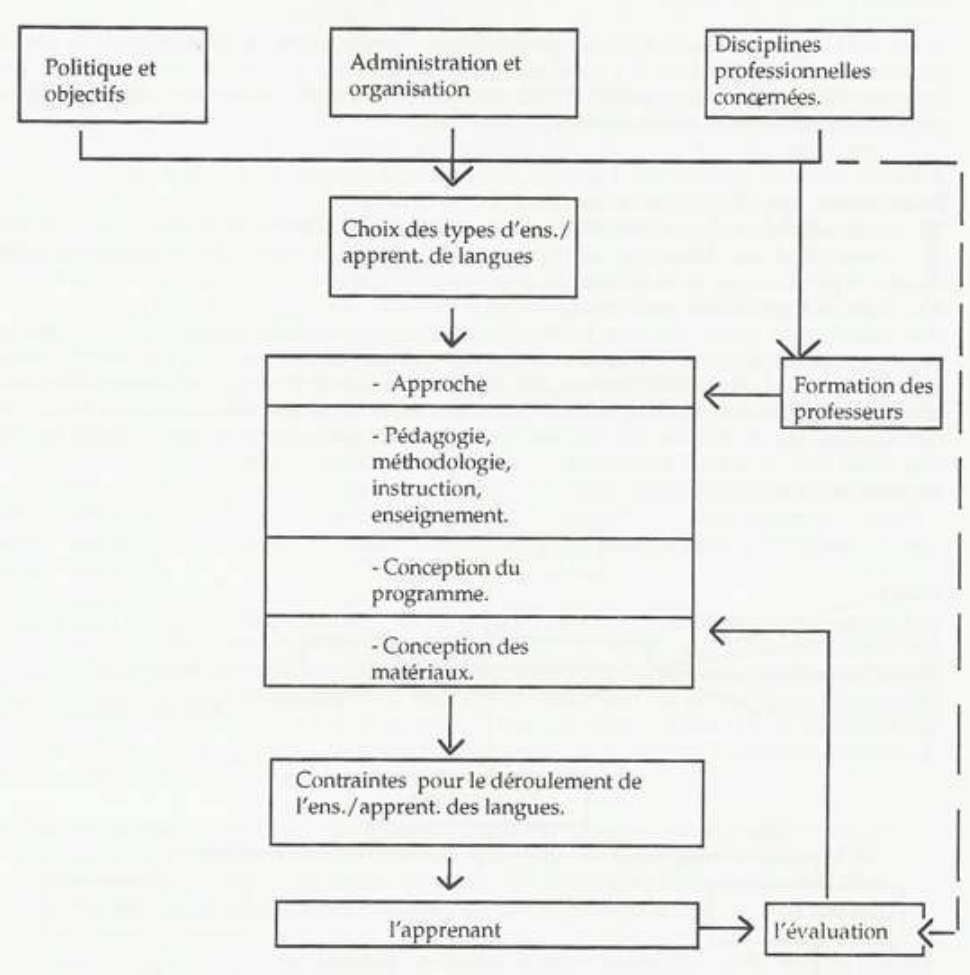

Strevens $1977: 35$

\section{orientations idéologi
disciplines connexes.}

2) au cœur: "l'approche" (l'a priori théorique qui conditionne les grands choix méthodologiques), la dimension méthodologique de l'enseignement, la conception du programme et l'élaboration des matériaux regroupés dans un même cadre qui représente le domaine relevant strictement de la responsabilité du professeur.

31 3) en aval : les contraintes liées au projet éducatif en matière de programmation (durée et périodicité), de compétence de l'enseignant, etc. En dernière position seulement apparaissent les apprenants qui seraient pourtant chacun individuellement redevables, dans l'idéal, d'un programme particulier, comme il sera vu plus loin.

\section{Démarches spécifiques aux langues de spécialité}

Le modèle d'organisation spécialement adapté aux LDS qu'offre Munby (1978) est un exemple d'une dimension supplémentaire donnée à la palette des critères à prendre en compte. Il s'agit de la dimension psychosociologique qui s'ajoute à toutes les exigences découlant de l'orientation communicationnelle du cours. Ce modèle est sans nul doute 
l'un des plus complets du genre. Son originalité tient au concept de Communicative Needs Processor (gestionnaire des besoins communicationnels ?) qui est l'élément central qui intègre tous les paramètres psychosociologiques tenant à la personnalité de l'apprenant, son environnement affectif et cognitif (cf. Bloom 1956, 1964), les modes de formulation des items linguistiques nécessaires, etc. L'accent est mis sur les situations langagières et les interactions dans lesquelles interviendra l'apprenant. Cette strate s'ajoute ou conditionne celle des formes linguistiques à utiliser.

Modèle pour la spécification de la compétence communicationnelle

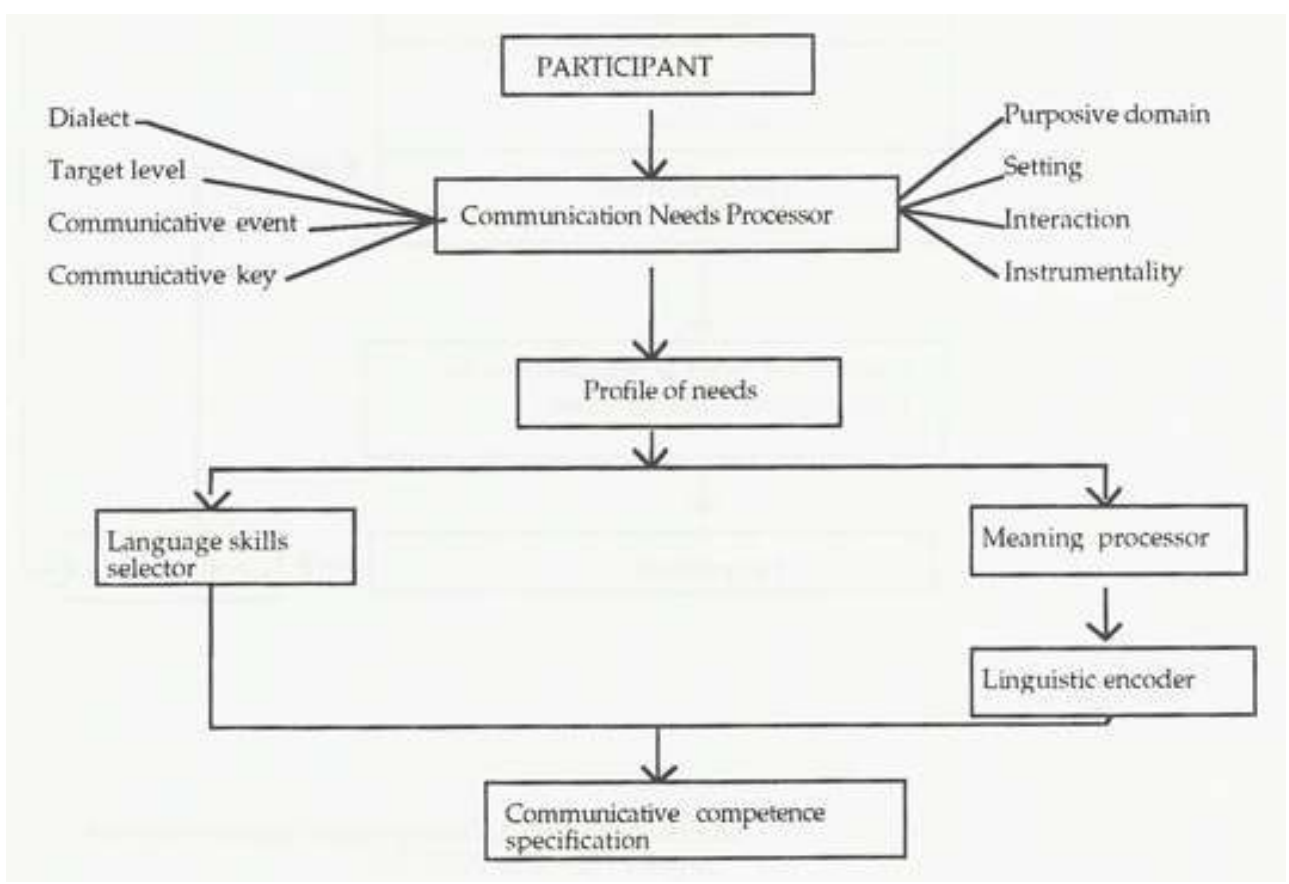

Munby 1978

D’autres strates sont représentées par les modes de communication qui seront utilisés (écrit ou oral), les "événements communicationnels» (communicative events): les fonctions langagières qui seront conditionnées par les situations langagières, etc. Les manifestations linguistiques qui caractériseront ces besoins devront encore être filtrées par différents systèmes de codage linguistiques et sémantiques. On le comprend aisément, ce modèle avait peu de chance de trouver une application directe et pratique à la conception d'un programme d'enseignement en LDS et n'a été utilisé que sélectivement ou comme référence théorique par certains praticiens de LDS.

D'autre part, comme le font remarquer Hutchinson et Waters (1987), « en menant l'analyse des besoins cibles à sa conclusion logique (Munby) a démontré l'inutilité finale d'une démarche d'analyse des besoins centrée sur la langue» (notre traduction). Hutchinson et Waters jugent essentiels les besoins d'apprentissage : la façon dont on apprend à utiliser une langue. L'analyse des besoins doit, pour eux, être centrée sur les processus d'apprentissage.

La même opinion a été formulée par Widdowson (1983 : 52) qui s'oppose au classement répertorié des aptitudes (formes ou fonctions langagières) en tant qu'items isolés, ce qui équivaut à nier leur caractère d'interdépendance dynamique et la hiérarchie qui existe entre eux. Certaines aptitudes relèvent de simples automatismes comme l'articulation des 
sons dans des mots isolés tandis que d'autres relèvent d'un ordre supérieur comme, par exemple, la compréhension de concepts ou celle des différentes parties d'un texte grâce aux marqueurs de cohésion lexicaux.

\section{Programmes spécifiques ou programmes généraux ?}

Est-il possible de proposer une démarche organisationnelle générale commune à l'ensemble des différentes LDS qui soit suffisamment souple pour tenir compte de leurs spécificités multiples (présentes et futures) et suffisamment structurée pour jouer le rôle de guide que l'on attend de tout programme d'enseignement?

Il semblerait que, jusqu'à présent, tout programme, ou toute stratégie organisationnelle en LDS, soit conditionné par la spécificité de l'objectif final à atteindre ou de la langue spécialisée à acquérir. Un modèle spécifique à l'anglais de spécialité est proposé par Mackay (1980) (voir page suivante).

Ce modèle était spécifiquement destiné à préparer des élèves vétérinaires mexicains à lire une documentation professionnelle rédigée en anglais. Il était donc nécessaire de définir très précisément les aptitudes opérationnelles et le type de langue à acquérir ainsi que de dégager une méthodologie unique pour l'enseigner. Le concept de spécificité est ici suffisant pour rendre différente et particulière la forme d'un modèle d'enseignement. Mais il n'en demeure pas moins que les objectifs pédagogiques pour y parvenir, et non plus seulement terminaux, doivent être définis. C'est ce qu'exprime Widdowson (1983: 90) :

The shift towards specificity will generally mean that purpose is conceived of as the acquiring of a limited competence, and the schematic (= conceptual) content of the course will then approximate more closely to the language of eventual aims. But there will still be the necessity of establishing pedagogic objectives.

Ces objectifs pédagogiques, que Mackay nomme "special methods", sont définis par une méthodologie spécifique liée aux aptitudes et langue à acquérir. En fait, tout programme d'enseignement en LDS a été jusqu'à présent conditionné par la spécificité des objectifs à atteindre: il semble qu'il ne puisse exister, dans ce domaine, que des modèles et des programmes spécifiques.

S'il existe une démarche organisationnelle commune à l'ensemble des différentes LDS qui constituerait un noyau commun à partir duquel l'on pourrait particulariser tel ou tel cheminement en fonction de telle ou telle spécificité, il faudrait sans doute, pour la trouver, se tourner du côté de l'apprenant.

Le rôle de celui-ci dans le processus d'enseignement devrait être éminemment actif et le cours devrait être conçu de telle sorte que la plupart des activités offrent l'occasion à l'apprenant d'adopter une démarche cognitive. La méthodologie du cours permettrait aux apprenants de sélectionner parmi les activités pédagogiques proposées celles qui seraient susceptibles d'être adaptées et transposées ultérieurement en fonction des besoins qui seraient les leurs au-delà de la période d'apprentissage. C'est ce que Widdowson (ibid.: 90) nomme des « activités procédurales appropriées »:

This means the devising of a methodology to involve the learners in appropriate procedural activities which will enable them to engage their capacity and realize the schematic content of the course to achieve the competence they need and the ability to exploit it subsequently in actual instances of language use. 
Fig. 2 Modèle de procédure pour la conception d'un programme d'anglais de spécialité

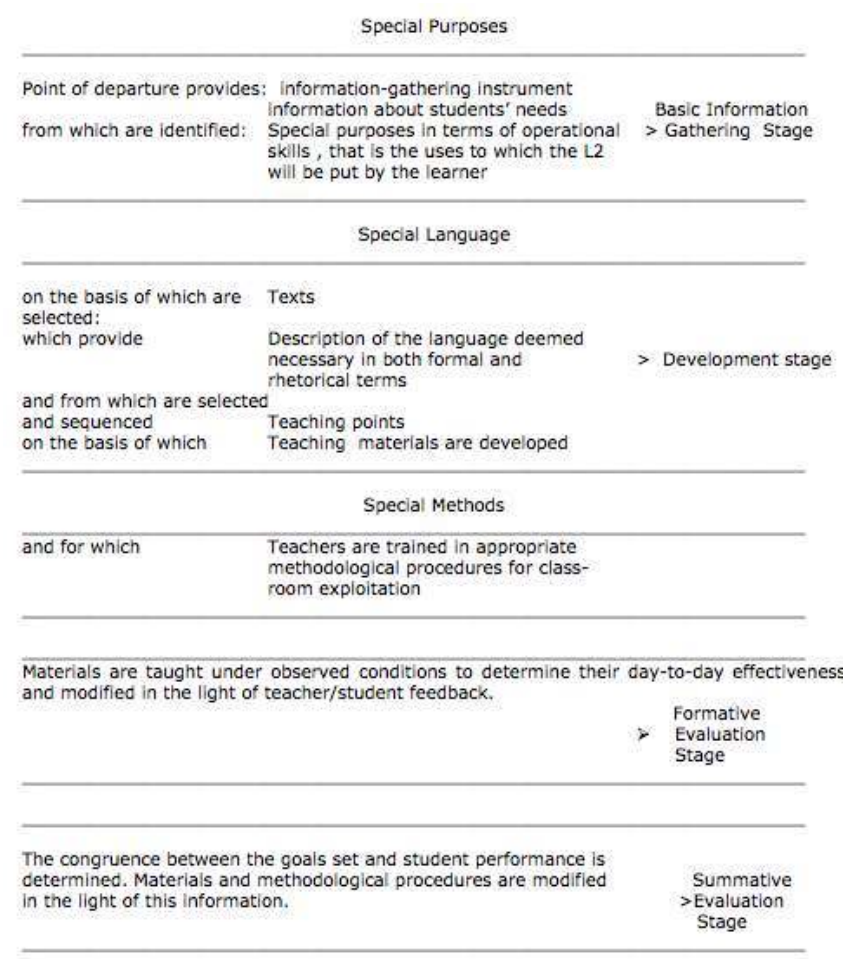

Mackay 1980

Un corollaire au rôle essentiel joué par les "activités procédurales » est l'importance désormais relative du contenu linguistique spécifique du programme. Le but recherché est, en effet, la capacité à transposer une attitude vis-à-vis d'une situation langagière plutôt que de pouvoir reproduire dans la vie réelle, de manière identique et en tenant compte de tous les éléments du discours (linguistiques, comportementaux, sémantiques, etc.) une situation d'apprentissage. C'est ce qu'exprime Crocker (1981:8) :

... topic itself is not as important as attitude towards content. That is, a specific purpose course does not view information about language as being sufficient content in itself, and the emphasis is more one of deriving information about language use or usage from the topic than of using a topic primarily as a way of exemplifying aspects of language. What this in turn suggests is that the focus of interest in an LSP course is on implementation.

La capacité à «mettre en œuvre » semble être la pierre de touche de la pertinence ou de l'inadaptation du cours de LDS :

Since there is no compulsion to set LSP course objectives in terms of language, there are no constraints on the methodology of an LSP course other than what works. (Crocker $1981: 15)$

Si l'on poursuit dans cette direction, il s'agit de savoir si l'on souhaite que le programme définisse l'enseignement comme un produit (on ne se préoccupe que du résultat immédiat d'une formation en fonction d'objectifs très précis et limités) ou bien comme un processus (l'enseignement va au-delà de l'entraînement à des besoins immédiats et envisage la préparation et l'adaptation à d'autres besoins plus généraux ou diversifiés). Widdowson considère que, par exemple, l'anglais de spécialité n'a pas d'existence propre et ne peut se justifier qu'en fonction de la science ou de la technique à laquelle il est 
associé. Il pousse donc le raisonnement jusqu'à nier l'utilité d'un programme spécifique pour les LDS et prône l'adoption du même modèle d'enseignement et de la même méthodologie que ceux des domaines d'application de la langue.

... to design (...) programmes by direct reference to the methodologies of subjects concerned on the grounds that these must of their nature incorporate the cognitive styles associated with their particular areas of study. (Widdowson 1984 : 186)

Parmi les arguments plaidant contre l'existence d'un programme (outre la position de Widdowson) pourrait se trouver l'inévitable décalage que l'on ne manquerait pas de souligner entre ce que l'on souhaite enseigner et ce qui sera réellement appris en fin de programme. Le deuxième argument serait qu'aucun programme, si complet soit-il, ne peut tenir compte de facteurs aussi subjectifs et abstraits que les émotions, les personnalités, la motivation, etc. Le troisième argument serait que tout programme ne peut s'adresser qu'à un "apprenant-robot", dans le sens où cette notion idéale d'apprenant type n'est en fait que la description de l'apprenant moyen, une minorité parmi d'autres au sein d'un groupe ou d'une classe.

Parmi les arguments en faveur de l'existence d'un programme en LDS, il faut citer l'obligation que fait un programme à son concepteur d'établir une hiérarchie parmi des objectifs, la nécessaire division du contenu d'un cours en segments enseignables, les limites mises au projet éducatif et la désignation d'objectifs finaux, la valeur de contrat que revêt un programme vis-à-vis des commanditaires et des apprenants, etc.

On le voit, les raisons militant pour l'existence d'un programme contrebalancent celles qui s'y opposeraient. Si l'on étudie les nombreuses méthodes commerciales existant sur le marché dans le domaine de l'anglais des sciences et des techniques, une LDS fortement représentée, on note que les modèles d'enseignement qui les sous-tendent ont en commun une orientation résolument communicationnelle et associent l'enseignement de formes linguistiques et de fonctions langagières à des situations professionnelles liées à des domaines particuliers d'application.

Cette trilogie formes linguistiques - fonctions langagières - situations professionnelles se justifie aux yeux des auteurs par l'argument selon lequel tout ce que le programme prévoit doit être d'un intérêt direct pour l'apprenant dans des situations qu'il rencontrera de manière prévisible. Les critères notionnels fonctionnels souvent employés ne peuvent cependant, à eux seuls, garantir que les objectifs communicationnels du cours seront atteints.

D'autre part, le fait même de prescrire des formulations et des formes correspondant strictement à des situations ou des besoins particuliers peut être considéré comme opposé au concept même de communication. Au-delà des programmes affichés par les auteurs (ce que Dakin, 1973, nomme "the external syllabus») existe un programme propre à chaque apprenant (“the learner's internal syllabus", Dakin, ibid.) et la difficulté est de faire coïncider le programme extérieur du professeur et les nombreux programmes intérieurs présents dans chaque groupe ou classe.

O’Neill (1977) préconise les trois mesures suivantes :

1) in the external syllabus we can concentrate at first upon those forms we know to be essential building blocks of the system and which are also of high, general communicative application (realising a variety of functions).

2) we can include outside this, either in separate distinct parts of our lessons or materials, or generally distributed within them, a variety of forms and lexis as well as techniques for understanding them which may be absorbed by different 
individual learners at different times according to the needs of their internal syllabuses.

3) we can keep returning constantly to the basic forms in the external syllabus and with less intensity to the various forms in the internal syllabuses.

La dernière mesure est le principe de l'enseignement concentrique et cyclique propre à l'approche notionnelle fonctionnelle : revenir fréquemment, sous des formes différentes et à l'occasion de thèmes différents, à la même forme linguistique devant être acquise. Toutefois, la complexité de l'acte de communiquer fait qu'un programme ne peut être conçu en fonction d'un seul paramètre (fonction, structure, etc.). Comme l'écrit Swan (1985) :

The real issue is not which syllabus to put first: it is how to integrate eight or so syllabuses (functional, notional, situational, topic, phonological, lexical, structural, skills) into a sensible teaching programme.

51 Un dernier élément est à verser au dossier du bien-fondé ou des inconvénients de disposer d'un programme en LDS, il s'agit du danger qu'il y aurait dans le cadre institutionnel d'un établissement, à élaborer un programme trop rigide ou contraignant, parce qu'orienté, par exemple, exclusivement vers une évaluation finale. Comme le soulignent Curiel et Murray (1984) :

It is of crucial importance that the course have the potential to be altered (....) according to the students' responses (needs) so that gradually the course evolves to reflect not the teacher's assumptions, but what is truly interesting to the students.

Certains praticiens de LDS pensent que pour que la trilogie formes linguistiques fonctions langagières - situations professionnelles puisse devenir une stratégie d'enseignement, il convient de réduire au maximum la difficulté que peuvent représenter les concepts scientifiques ou techniques contenus dans les matériaux d'enseignement. Elliott (1976) décrit cette situation de la manière suivante :

If the student is distracted by the need to concentrate on the relationship or process being described, one of two things will happen. He will mentally translate to ease the language burden, or he will concentrate on getting the language point right while totally ignoring the meaning. Both of these reactions are inimical to the development of fluency.

Ce praticien a donc décidé de considérer le texte authentique comme incluant des "lexical slots», cases lexicales renfermant des notions scientifiques et techniques complexes, dont le contenu peut être modifié pour inclure des notions plus simples qui appartiennent à la vie quotidienne et à la langue générale. Le but est de permettre à l'apprenant de se concentrer sur les formes linguistiques et les fonctions langagières en allégeant la charge cognitive que peut représenter la compréhension des situations et notions professionnelles (voir annexe).

Il s'agit ici d'une option pédagogique particulière qui n'a pas manqué en son temps de diviser les enseignants de LDS ; pourtant c'est l'exercice de transposition d'un lexique, ou d'un contexte, à un autre que met en œuvre une forme discursive (narration, interpellation, questionnement, etc.) jugée utile à acquérir, qu'il faut, selon nous, retenir comme démarche organisationnelle générale du cours de LDS. 


\section{Démarche 'procédurale' adaptée aux langues de spécialités} (edagogique bien connu qu'est le " déficit d'information » (" information gap ») qui oblige les apprenants à dialoguer entre eux et avec le professeur et à négocier le sens de leurs productions, mais aussi au x «déficits de raisonnement » ou « d'opinion » (« reasoning or opinion gaps ») qui contraignent les apprenants à prendre position sur des concepts ou des idées qu'ils doivent dénoncer ou défendre.

62 5) La facilité de gradation d'un programme qui dépend, non plus d'une hiérarchie de difficulté supposée entre des formes linguistiques, mais de la gradation des tâches ellesmêmes.

63 6) L'aspect nécessairement répétitif de micro-tâches comme devoir se présenter oralement avant une simulation ou un jeu de rôle, devoir préparer un court texte qui servira de compte-rendu publié dans le journal des élèves à la suite d'une tâche complexe menée à bien par un groupe, etc.

7) L'extrême variété des tâches qui, si elles ont été judicieusement choisies, peuvent relever de contextes très différents et néanmoins préparer à l'accomplissement de tâches langagières qui relèvent strictement du domaine d'application professionnel ou académique de l'apprenant. Ces dernières tâches ont été identifiées à la suite d'une analyse des besoins très détaillée qui a été fournie, une fois pour toutes, à l'apprenant en début d'année. 
En résumé, la démarche procédurale ne conçoit le programme de formation en LDS non plus linéairement mais atomiquement autour de quelques tâches ou noyaux d'activités à forte potentialité de transfert vers d'autres contextes plus spécifiques et spécialisés. Ces noyaux d'activités peuvent relever de la langue générale ou de domaines d'application spécialisés autres que ceux des apprenants.

Cette démarche ne prévoit volontairement pas tout ce qui peut se passer dans le cours ; elle ne le pourrait d'ailleurs pas. De même, le professeur ne contrôle pas complètement l'évolution du cours qui fait, évidemment et par nature, une place importante au programme interne de l'apprenant.

En guise de conclusion, nous livrons ici un exemple concret de programme bâti autour de tâches qui vont être proposées en 1993-94 à des élèves ingénieurs de $2^{\mathrm{e}}$ année de l'École Centrale de Nantes spécialisés en automatique, productique et calcul des structures. Ce programme couvrira environ 70 heures de cours et regroupera une dizaine de tâches. Les trois tâches détaillées plus bas feront l'objet d'environ 25 heures d'activités en cours et d'environ 10 heures de travail périphérique de préparation (voir tableau 2).

Tableau 2

\begin{tabular}{|c|c|}
\hline TÂCHE PRINCIPALE & TÂCHES ANNEXES OU INDUITES \\
\hline $\begin{array}{l}\text { - Rédaction en groupe d'une notice technique } \\
\text { accompagnant un lecteur CD (le point de départ étant une } \\
\text { notice "surréaliste" proposée par le constructeur } \\
\text { taiwanais) }\end{array}$ & $\begin{array}{l}\text { - Lettre de protestation adressée au } \\
\text { constructeur. } \\
\text { - Refus d'application de la garantie } \\
\text { opposé par le vendeur qui défend } \\
\text { les documents d'origine. (oral et } \\
\text { écrit) }\end{array}$ \\
\hline $\begin{array}{l}\text { - Réunion des membres d'un bureau d'études au sujet de } \\
\text { la conception d'un modèle évolué de pupitre de } \\
\text { conférencier. À l'issue de } 2 \mathrm{~h} 30 \text { de réunion, un nouveau } \\
\text { concept industriel est présenté oralement, par écrit et à } \\
\text { l'aide de schémas par le groupe. }\end{array}$ & $\begin{array}{l}\text { - Discussion des modalités de dépôt } \\
\text { d'un brevet (participation d'un } \\
\text { expert extérieur spécialisé, lui aussi } \\
\text { anglophone) } \\
\text { - Projet de programme vidéo de } \\
\text { présentation en 3-D (+ création d'un } \\
\text { logiciel de présentation) } \\
\text { - Prospection de sponsors } \\
\text { étrangers. }\end{array}$ \\
\hline $\begin{array}{l}\text { - Construction d'un avion en papier à partir des mêmes } \\
\text { matériaux par les apprenants travaillant en binômes. }\end{array}$ & $\begin{array}{l}\text {-Rédaction d'un cahier des charges. } \\
\text {-Enregistrement vidéo avec } \\
\text { commentaire en direct des essais en } \\
\text { vol. } \\
\text { - Analyse écrite du comportement } \\
\text { des prototypes et suggestions } \\
\text { d'amélioration } \\
\text { - Constitution d'une commission } \\
\text { technique d'examen des différents } \\
\text { soumissionnaires. }\end{array}$ \\
\hline
\end{tabular}


En fonction du temps disponible, les tâches principales de $2^{\mathrm{e}}$ et $3^{\mathrm{e}}$ trimestres seront choisies parmi la liste suivante :

- La mise au point d'un système d'échange étudiants/étudiants ou étudiants/chercheurs avec un pays anglophone.

- La complexification du jeu de dames.

- L'organisation des « 24 heures - Solex» de l'école avec parrainage exclusif de sociétés étrangères.

- La production d'un document de travail organisant concrètement l'introduction d'une $2^{\mathrm{e}}$ langue étrangère obligatoire dans l'emploi du temps avec nécessité de réaménager certaines activités de l'école.

- La transformation de l'école en usine à cigares avec nécessité d'utiliser au mieux toutes les installations existantes et les personnels. Plans et schémas d'exploitation à fournir.

- Le recrutement d'un nouveau professeur de langues au sein d'une équipe déjà formée (simulation d'embauche)

- Doublage d'un épisode de Chapeau melon et bottes de cuir à partir d'une version vidéo muette ou française.

\section{BIBLIOGRAPHIE}

Bloom, B.S. (dir.). 1956. Taxonomy of Educational Objectives: Cognitive Domain. Londres : Longman.

Bloom, B.S. (dir.). 1964. Taxonomy of Educational Objectives: Affective Domain. Londres : Longman.

Crocker, T. 1981. « Scenes of endless science: ESP and education ». ELT Doc. 112. « The ESP

Teacher: Role, Development and Prospects ». Londres : The British Council.

Curiel, D.N. \& I.P. Murray. 1984. « A rationale for materials production in ESP ». English Teaching Forum 22/ 2.

Dakin, J. 1973. The Language Laboratory and Language Learning. Londres : Longman.

Elliott,C.R. 1976. « Must scientific English be dull ?». English Language Teaching Journal 21/1, 29-34.

Hutchinson, T. \& A. Waters. 1980. « ESP at the crossroads ». English for Specific Purposes 36, Oregon State University, 1980.

Hutchinson, T. \& A. Waters. 1987. English for Specific Purposes, A Learning-centred Approach. Cambridge : Cambridge University Press.

Mackay, R. 1980. «English for Specific Purposes: a Mexican Case-study ». English Teaching Forum $18 / 2,8-12$.

Mackey, W.F. 1965. Language Teaching Analysis. Londres : Longmans, Green and Co. Ltd.

Munby, J. 1978. Communicative Syllabus Design, a Sociolinguistic Model for Defining the Content of Purpose Specific language Programmes. Cambridge : Cambridge University Press.

O'Neill, R. 1977. « The limits of functional-notional syllabuses, or 'My guinea-pig died with its legs crossed' ». In Holden, S. (dir.), English for Specific Purposes. Londres : Modern English Publications. 
Prabhu, N.S. 1987. Second Language Pedagogy. Oxford : Oxford University Press.

Strevens, P. 1977. New Orientations in the Teaching of English. Oxford : Oxford University Press.

Swan, M. 1985. « A critical look at the communicative approach (2). » English Language Teaching Journal 39/2.

Widdowson, H.G. 1983. Learning Purpose and Language Use. Oxford : Oxford University Press.

Widdowson, H.G. 1984. Explorations in Applied Linguistics (2). Oxford : Oxford University Press.

\section{ANNEXES}

Texte authentique provenant du domaine de la chimie :

In compounds such as water, alcohols, amines, carboxylic acids, and others which possess both (1) a hydrogen attached to oxygen or nitrogen, and (2) a donor atom such as oxygen or nitrogen, intermolecular proton exchange can occur. The hydrogen atom possesses a certain positive character, relative to the other atom, which may be described by the symbol (symbol given). The result of this is that a certain degree of organization exists in a medium consisting of such molecules, brought about by a weak binding, largely electrostatic in nature, caused by the attraction of positive and negative ends of the dipoles." (Geissman, T.A. Principles of Organic Chemistry. Freeman and Co., San Francisco, 1959, p. 66)

Texte ré-crit dans lequel les difficultés conceptuelles ont été réduites par transposition du lexique scientifique :

With people such as actors, composers, and artists who possess both (1) a creative ability linked with expressiveness or exhibitionist tendencies, and (2) an opportunity such as a party or a show, rather amusing exchanges can occur. The creative person possesses a more forceful character relative to other people. The result of this is that a certain degree of antagonism exists in a group consisting of such people, brought about by inflexibility, largely eccentric in nature, caused by a refusal to listen to others. (Elliott, 1976: 32)

\section{NOTES}

1. Dorénavant LDS, dont les deux formes majoritaires pourraient être siglées LFP pour les langues à finalité professionnelle et LEE pour celles destinées à permettre des études à l'étranger.

\section{RÉSUMÉS}

L'article traite des différents modèles et programmes d'enseignement appliqués à des langues de spécialité (LDS) depuis une trentaine d'années. Il s'agit de modèles originellement destinés à l'enseignement des langues générales adoptés tels quels pour les LDS, puis de modèles transitoires ou hybrides qui font une place à la spécificité des objectifs en LDS, enfin de modèles 
spécifiquement conçus pour les LDS. L'évolution de la notion de modèles et de programmes pédagogiques centrés sur l'apprenant et la nécessité de susciter et d'entretenir sa motivation aboutissent à une proposition d'organisation du cours de LDS reposant sur une série de tâches graduées. Ces tâches représentent des noyaux d'activités de tronc commun à fort potentiel de transfert ultérieur vers les domaines d'application spécifiques des apprenants.

This article deals with the different teaching/learning models and syllabuses applied to Languages for Specific Purposes (LSP) over the last thirty years. The early models originally destined for General Purpose Languages were applied unchanged to LSP. Eventually, hybrid or transition models which allowed for the specificity of LSP objectives were developed. The next stage saw the implementation of syllabuses specifically designed for LSP. The notion of learnercentred syllabus models and the necessity to create and maintain motivation led the present author to suggest the organization of task-based LSP courses. The tasks involved are commoncore activity clusters with high potential for later transfer to the learners' domains of specific application.

\section{INDEX}

Keywords : language for specific purposes, pedagogy, procedural approach, model, syllabus design, task-based approach

Mots-clés : démarche procédurale, langue de spécialité, modèle, pédagogie, programme d'enseignement, tâche

\section{AUTEUR}

\section{CLAUDE SIONIS}

Claude Sionis enseigne à l'École Centrale de Nantes. claude.sionis@wanadoo.fr 